\title{
Use of a hardware-software complex for phytosanitary monitoring and chemical treatments of the Sosnowski hogweed
}

\author{
Timur Kornilov ${ }^{1}$, Anton Terentev ${ }^{2 *}$ and Valeriy Kekelidze ${ }^{3}$ \\ ${ }^{1}$ All-Russian Institute of Plant Protection, 196608, St.Petersburg, Pushkin, Podbelskogo 3, Russian \\ Federation \\ ${ }^{2}$ Tyumen State University, 625003, Tyumen, 23 Lenina St., Russian Federation \\ ${ }^{3}$ 2050.Digital LLC, 125009, Moscow, Bolshoy Gnezdnikovsky per. 1 bld 2, Russian Federation
}

\begin{abstract}
A new technology for the detection and chemical treatment of the Sosnowski hogweed using neural networks and ground and air robotic systems was tested. Confirmation is obtained of the possibility of detecting invasive weeds and their state using satellite imagery and UAVs. The features of the chemical treatment of dicotyledonous plants using UAVs are investigated. The effectiveness of the weed control by chemical pesticides sprayed with UAVs was confirmed by the example of the "Graund" herbicide.
\end{abstract}

\section{Introduction}

The Sosnowski hogweed is an invasive species of plants of the Umbelliferae family, the initial distributional range of which is the Central and Eastern Caucasus. In the USSR, it was originally intended for using as a promising crop for obtaining a forage base for cattle. However, serious shortcomings were soon revealed: the deterioration in the quality of meat and dairy products, as well as various violations of the reproduction of offspring. It turned out that the juice of hogweed contains photosensitizing substances, which, on contact with human skin, can cause severe burns, and the sun's rays significantly increase its effect. When contacting the eyes, hogweed juice can lead to blindness. There are various allergic reactions to the inhalation of hogweed pollen up to death as a result of asphyxiation $[1,2]$.

The use of various digital technologies in agriculture has recently become increasingly spread around the world. Most widely, such technologies are used in monitoring plant diseases and pests, including detection of invasive plants, monitoring the state of field crops and yield, forecasting yield and also accurately applying fertilizers, water and plant protection products $[3,4]$. The worldwide practice is the use of Big Data technologies, neural networks, digital field doubles and various data from UAVs and satellites to obtain detailed information about the field status online $[5,6]$.

This work is devoted to the joint development of a method for the Sosnowski hogweed control, sponsored by 2050.Digital LLC and the All-Russian Institute of Plant Protection.

\footnotetext{
*Corresponding author: admin@vizr.spb.ru
} 
As a part of the work, research was conducted on the development of a hardware-software complex for monitoring and further chemical treatment of the Sosnowski hogweed. The advantage of the method is the use of neural networks for the recognition of processing objects, the automatic creation of UAV tracks, program control at all stages of weed control and processing, mainly using ground and air robotic devices, including using ultra lowvolume spraying technologies.

\section{Material and methods}

According to the developed technology, a preliminary analysis of the distribution of the Sosnowski hogweed, if necessary, is based on satellite imagery using spectral analysis. This step is not obligatory, since in most cases hogweed clusters can be localized based on the results of standard monitoring methods. In the case of this study, such an analysis was not performed, since the exact area of work was determined in advance.

The next step is to create flight routes for aircraft type UAV reconnaissance. Their goal is to confirm the clusters and identify individual foci of distribution of the Sosnowski hogweed. Based on the obtained data, the neural network developed by 2050.Digital LLC creates a processing map in which there are allocated zones and tracks for processing using ground-based spraying robots, spraying drones, as well as manual ground-processing zones. Usually, these tracks are created in accordance with the data of geographic information systems in a given area, which automatically avoids errors associated with violation of environmental and sanitary standards.

Ground robots are boom sprayers of increased cross-country ability on a caterpillar chassis, equipped with an automatic control and GPS positioning systems and are used on vast flat areas. Their speed is similar to the speed of a standard ground sprayer. In the case of focal distribution of the Sosnowski hogweed, the precision method of herbicide spraying is used instead of boom spraying. Spraying drones are helicopter type UAVs with a hanging tank capable of carrying 10 litres of herbicides and are used for processing limited or inaccessible areas. Ground manual processing is used in local areas where processing by ground robots and drones is impossible or inappropriate. After thenecessary period of time, the efficiency is monitored using UAV reconnaissance aircraft equipped with an intelligent recognition system of the Sosnowski hogweed in various state, namely damaged, intact, partially damaged and new shoots of the plants.

Various herbicide compositions have been developed and tested taking into account the seasonality of treatment (May-June and September). The compositions of herbicidal mixtures possess both the ability to destroy actively vegetating plants and create a protective soil screen to prevent new shoots in the period after treatment. Preference is given to selective herbicides that destroy dicotyledonous broad-leaved weeds (including hogweed), but leave untouched the turf of monocotyledonous grasses. This strategy aims to ensure that dense turf prevents the germination of hogweed seeds that are left in the soil from past seasons.

On May 28, 2019, work was carried out to develop the application of herbicides against the Sosnowski hogweed using a quadrocopter on the territory of the Naro-Fominsk city district of the Moscow region on a land plot with cadastral number 50: 26: 0130801: 88 owned by OAO Voskhod (OGRN 1155030000476, TIN 5030084864, gearbox 503001001). As a control experiment, the treatment was carried out using a backpack sprayer with the same herbicides with similar application rates. Weather conditions were as follows: air temperature 26-28 degrees Celsius, air humidity $42 \%$ with a decrease to $37 \%$, wind speed 2.4-3.6 m / s. The experimental plots were a field with various wild plants, with focal patches of the Sosnowski hogweed from 2 to 30 square meters in the phase of 5-6 true leaves. 
In preparation for processing, an optimum flight altitude of 2.5 - 4 meters was determined for the aircraft from the point of view of ensuring flight safety and the effective application of pesticides. The actual strip width of the treated surface which provides sufficient application efficiency was determined when operating at given flight altitudes and wind speed using an UAV quadrocopter, which is from 1.8 to 2.5 meters, depending on the conditions. The capacity of the UAV's tank for the pesticides solution was 10 litres.

When conducting the experiments on the Sosnowski hogweed control using a helicopter type UAV as a carrier of spraying equipment, special water-sensitive cards measuring 26 by 26 millimetres were used to determine the effective spraying width and the number of drops of deposited liquid per unit of the treated area. Numbered water-sensitive cards were laid out on artificial supports at a height of 30 centimetres from the surface of the earth perpendicular to the direction of UAV movement when spraying in increments of 0.5 meters. The evaluation of the width of effective spraying was carried out in three replications. Quantitative assessment was carried out visually by counting the number of drops per 1 square centimetre using Adobe Photoshop after scanning the cards. According to the results of the experiments, it can be said that the real width of effective spraying in the given conditions was from 180 to 220 centimetres with a flight height of $2.5-3$ meters and an UAV moving speed of 3 meters per second.

The experimental plot was processed with herbicides at different application rates : "Ankor ${ }^{\circledR-85 " ~(a c t i v e ~ i n g r e d i e n t: ~ s u l f o m e t u r o n-m e t h y l) ~ a n d ~ " G r a u n d " ~(a c t i v e ~ i n g r e d i e n t: ~}$ glyphosate), according to the scheme developed by FSBSI VIZR and 2050.Digital LLC: 4 replicates, 3 concentration options for each preparation for application with an UAV. Control experiment with a backpack sprayer was carried out at the maximum concentration of both herbicides. According to their characteristics, the actual effectiveness was determined fourteen days after the treatment. The manufacturer's norms of application of the "Graund" solution are from 2 to 8 litres of herbicide containing 360 grams per litre of glyphosate per hectare, and the norms of application of the "Ankor ${ }^{\circledR}-85$ " solution are from 50 to 200 grams of herbicide containing 750 grams per kilogram of sulfometuron-methyl per hectare.

On June 11, 2019, the effectiveness of the herbicides treatment against the Sosnowski hogweed was evaluated. Accounting was carried out by visual inspection of damaged, intact, partially damaged plants and new seedlings of the Sosnowski hogweed. Calculations were made separately for these categories of plants, afterwards the effectiveness of herbicides was calculated by formula (1):

$$
E f=\frac{N t-N i}{N i} * 100 \%
$$

$E f$ - Efficiency; $N t$ - the number of hogweed plants in the experiment, total; $N i$ - the number of intact hogweed plants in the experiment, total

\section{Results and discussion}

The purpose of this paper was to determine the effectiveness of the developed technology for the detection of the Sosnowski hogweed using remote sensing with various methods, such as satellite imaging and UAVs, as well as the use of herbicides against invasive plants using UAVs.

In the case of the herbicide "Graund" (active ingredient: glyphosate), we can conclude that the technology for the use of herbicides with UAVs, presented in these tests, is working and effective. This is confirmed by three experiments with four replicates in each experiment with different concentrations of the herbicide as the average efficiency for all 
experiments with different concentrations of the herbicide is around 99 percent. According to the results of the experiments, the herbicide "Graund" showed a high average efficiency in all experiments. The average efficiency for 4 experiments with three repetitions with the minimum recommended concentration of herbicide (2 liters per hectare, application with UAVs and a manual sprayer) was ninety-eight percent. In other experiments, also carried out with three replicates ( 4 and 8 liters per hectare), the effectiveness of the herbicide was one hundred percent.

Ankor- 85 herbicide showed an average efficiency of $73 \%$ at maximum possible concentrations recommended by the manufacturer of 200 grams of herbicide per hectare when using a backpack sprayer and $61.2 \%$ efficiency in a similar experiment when applied with UAVs, however, its effectiveness drops sharply when the concentration of the herbicide decreases (average efficiency is $41.5 \%$ at 100 grams per hectare when applied from an UAV and $22.1 \%$ at 50 grams per hectare when applied from an UAV).

When monitoring the Sosnovski hogweed using satellite imagery, the percentage of accurate determination of plant clusters by spectral characteristics was 85 percent. It is worth noting that during training of the neural network, the accuracy of determination increases as the database expands (the initial accuracy was sixty percent), and it is planned to reach ninety-nine percent accuracy over the next year and a half. When monitoring the Sosnovski hogweed using an UAV scout, the percentage of the determined hogweed plants of the total number of plants in the study area was ninety-nine percent in the complete absence of false detections. The percentage of coincidence of hogweed plant state estimates with visual control data when using a hardware-software complex for monitoring the Sosnovski hogweed was ninety-seven percent. It is planned that it will reach ninety-nine percent over the next year when additional data shall be collected for the neural network training.

Based on the results of the experiments, we can conclude that the technology for using a software and hardware complex for phytosanitary monitoring and chemical treatments of the Sosnowski hogweed is effective, both in monitoring and application of herbicides using UAVs.

\section{References}

1. N.N. Luneva, G.Yu. Konechnaya, T.N. Smekalova, I.G. Chukhina, Plant Protection News [Vestnik Zashchity Rasteniy] 3(97), 10-15 (2018) http://doi.org/10.31993/23086459-2018-3(97)-10-15

2. O. Jakubowicz, C. Zaba, G. Nowak, S. Jarmuda, R. Zaba, J.T. Marcinkowski, Ann Agr Env Med 19(2), 327-328 (2012)

3. J.P. Dash, M.S. Watt, T.S.H. Paul, et al. Remote Sens 11(15), 1812 (2019)

4. E. Dhivya, V. Durai Raj, S. Vishal, et al. Comput Electron Agr 155, 257-282 (2018)

5. J. Mullerova, J. Bruna, T. Bartalos, et al. Front Plant Sci 8, 887 (2017)

6. E.V. Tovstik, T.A. Adamovich, V.V. Rutman, et al. Theor Appl Ecol 2, 35-37 (2018) http://doi.org/10.25750/1995-4301-2018-2-035-037 